\title{
Getting a ride between cells
}

a sorting signal present in TGBp3 has a key role in cell-to-cell spread of potexviruses

Plant viruses spread from cell to cell through plasmodesmata, which are cytoplasmic tubes that allow exchange of macromolecules between cells and contain extensions (desmotubules) of the endoplasmic reticulum (ER). However, the molecular mechanisms of viral spread are poorly understood. Now, Wu et al.

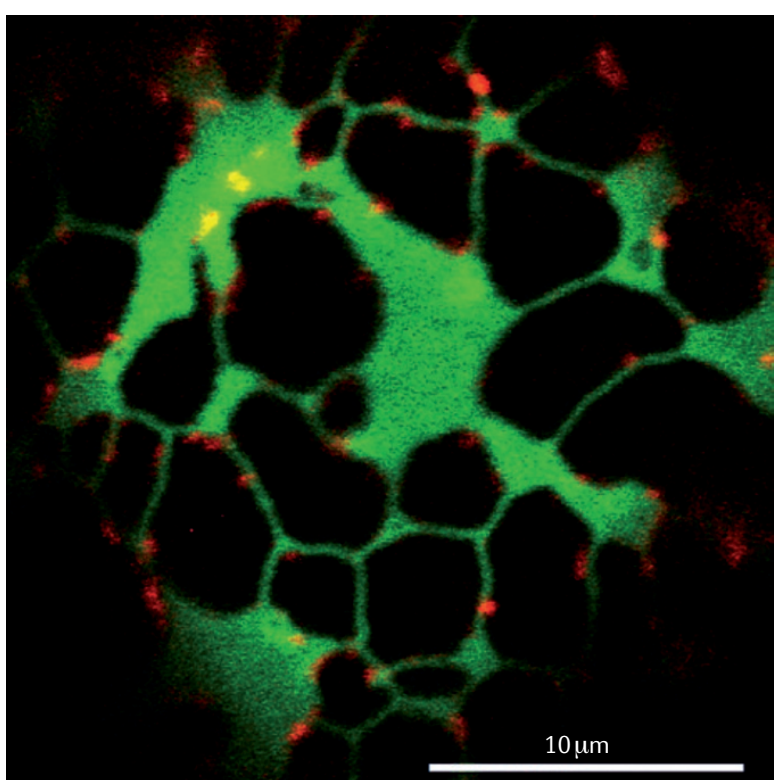

Peripheral view of a Nicotiana benthamiana leaf epidermal cell showing that triple gene block 3 protein (TGBp3) (fused to mCherry; red) is localized to tubules and curved areas of the endoplasmic reticulum (which is labelled with GFP-Arabidopsis thaliana SEC61 $\gamma$; green). Image courtesy of C.-H. Wu and C.-W. Wang, Academia Sinica, Taipei, Taiwan. report in The Journal of Cell Biology that a non-structural protein of potexviruses contains a sorting signal for the targeting of viral components to desmotubules during cell-to-cell viral transmission.

Many plant RNA viruses use non-structural proteins known as movement proteins for cell-to-cell viral spread. One of these movement proteins, triple gene block 3 protein (TGBp3) of bamboo mosaic virus (BaMV), is an integral membrane protein that forms clusters in curved areas of the peripheral (cortical) ER when expressed in either plant cells or Saccharomyces cerevisiae. To better understand the role of TGBp3 in viral spread, the authors generated deletions and point mutations in TGBp3, and found that proteins with specific mutations at the carboxy-terminal region, or lacking this region, localized to the perinuclear ER and did not form clusters in S. cerevisiae, whereas the $\mathrm{C}$-terminal region was localized in the cytoplasm when expressed alone. Furthermore, fusion proteins in which the TGBp3 aminoterminal region, which contains a putative transmembrane domain, was replaced with various transmembrane domains (derived from other proteins) were found to localize to the cortical ER and form clusters. Thus, the TGBp3 N-terminal region targets the protein to the perinuclear $\mathrm{ER}$, whereas the $\mathrm{C}$-terminal region contains a sorting signal for targeting transmembrane domains to curved areas of the ER. Moreover, viruses harbouring mutated variants of TGBp3 produced smaller lesions in plants, if they produced lesions at all, and they were defective in cell-to-cell spread.

In addition, the authors showed that specific interactions occur between the BaMV proteins, and they proposed a model in which TGBp2 and TGBp3 form a complex in the ER to which the capsid protein, TGBp1 and the viral RNA then attach. The new complex may then move along the ER toward desmotubules within plasmodesmata.

Thus, a sorting signal present in TGBp3 has a key role in cell-to-cell spread of potexviruses, and BaMV seems to move through plasmodesmata in the form of ribonucleoprotein complexes rather than virions.

Cesar Sanchez

ORIGINAL RESEARCH PAPER Wu, C.-H., Lee, S.-C. \& Wang, C.-W. Viral protein targeting to the cortical endoplasmic reticulum is required for cell-cell spreading in plants. J. Cell Biol. 193. 521-535 (2011) 